\title{
Parameter Augmentation for Two Formulas
}

\author{
Caihuan Zhang \\ Department of Mathematics, DaLian University of Technology, \\ Dalian 116024, P. R. China \\ zhcaihuan@163.com
}

Submitted: Jun 5, 2006; Accepted: Nov 7, 2006; Published: Nov 17, 2006

Mathematics Subject Classifications: 33D15, 05A30

\begin{abstract}
In this paper, by using the q-exponential operator technique on the $q$-integral form of the Sears transformation formula and a Gasper $q$-integral formula, we obtain their generalizations.
\end{abstract}

\section{Notation}

In this paper, we follow the notation and terminology in ([4]). For a real or complex number $q(|q|<1)$. let

$$
(\lambda)_{\infty}=(\lambda ; q)_{\infty}=\prod_{n=1}^{\infty}\left(1-a q^{n-1}\right)
$$

and let $(\lambda: q)_{\mu}$ be defined by

$$
(\lambda)_{\mu}=(\lambda ; q)_{\mu}=\frac{(\lambda ; q)_{\infty}}{\left(\lambda q^{\mu} ; q\right)_{\infty}}
$$

for arbitrary parameters $\lambda$ and $\mu$, so that

$$
(\lambda)_{n}=(\lambda ; q)_{n}=\left\{\begin{array}{lc}
1, & n=0 \\
(1-\lambda)(1-\lambda q) \ldots\left(1-\lambda q^{n-1}\right), & (n \in N=1,2,3, \cdots)
\end{array}\right.
$$

The q-binomial coefficient is defined by

$$
\left[\begin{array}{l}
n \\
k
\end{array}\right]=\frac{(q)_{n}}{(q)_{k}(q)_{n-k}}
$$

Further, recall the definition of basic hypergeometric series,

$$
{ }_{s} \phi_{s-1}\left[\begin{array}{c}
\alpha_{1}, \cdots, \alpha_{s} \\
\beta_{1}, \cdots, \beta_{s-1}
\end{array} \mid q ; z\right]:=\sum_{n=0}^{\infty} \frac{\left(\alpha_{1}, \cdots \alpha_{s}\right)_{n}}{\left(q, \beta_{1}, \cdots, \beta_{s-1}\right)_{n}} z^{n} .
$$


Here, we will frequently use the Cauchy identity and its special case ([4])

$$
\begin{gathered}
\frac{(a x ; q)_{\infty}}{(x ; q)_{\infty}}=\sum_{n=0}^{\infty} \frac{(a ; q)_{n} x^{n}}{(q ; q)_{n}} \\
\frac{1}{(x ; q)_{\infty}}=\sum_{n=0}^{\infty} \frac{x^{n}}{(q ; q)_{n}} \\
(-x ; q)_{\infty}=\sum_{n=0}^{\infty} \frac{q^{\left(\begin{array}{c}
n \\
2
\end{array}\right)} x^{n}}{(q ; q)_{n}}
\end{gathered}
$$

\section{The exponential operator $T\left(b D_{q}\right)$}

The usual q-differential operator, or q-derivative, is defined by

$$
D_{q}\{f(a)\}=\frac{f(a)-f(a q)}{a}
$$

By convention, $D_{q}^{0}$ is understood as the identity.

The Leibniz rule for $D_{q}$ is the following identity, which is a variation of the q-binomial theorem $([1])$

$$
D_{q}^{n}\{f(a) g(a)\}=\sum_{k=0}^{n} q^{k(k-n)}\left[\begin{array}{l}
n \\
k
\end{array}\right] D_{q}^{k}\{f(a)\} D_{q}^{n-k}\left\{g\left(q^{k} a\right)\right\}
$$

In ([3]), Chen and Liu construct a q-exponential operator based on this, denoted T:

$$
T\left(b D_{q}\right)=\sum_{n=0}^{\infty} \frac{\left(b D_{q}\right)^{n}}{(q ; q)_{n}}
$$

For $T\left(b d_{q}\right)$, there hold the following operator identities.

$$
\begin{gathered}
T\left(b D_{q}\right)\left\{\frac{1}{(a t ; q)_{\infty}}\right\}=\frac{1}{(a t, b t ; q)_{\infty}} \\
T\left(b D_{q}\right)\left\{\frac{1}{(a s, a t ; q)_{\infty}}\right\}=\frac{(a b s t ; q)_{\infty}}{(a s, a t, b s, b t ; q)_{\infty}}
\end{gathered}
$$

\section{A generalization of the q-integral form of the sears transformation}

In this section, we consider the following formula ( [3, Theorem 6.2])

$$
\int_{c}^{d} \frac{(q t / c, q t / d, a b c d e t ; q)_{\infty}}{(a t, b t, e t ; q)_{\infty}} d_{q} t=\frac{d(1-q)(q, d q / c, c / d, a b c d, b c d e, a c d e ; q)_{\infty}}{(a c, a d, b c, b d, c e, d e ; q)_{\infty}}
$$


Chen and Liu showed it can be derived from the Andrews-Askey integral by the qexponential operator techniques. Here, again using the q-exponential operator technique on it, we obtain a generalization of this identity. We have

Theorem 3.1. we have

$$
\begin{gathered}
\int_{c}^{d} \frac{(q t / c, q t / d, a b c d f t, b c d e f t ; q)_{\infty}}{(a t, b t, e t, f t ; q)_{\infty}} \times{ }_{3} \phi_{2}\left[\begin{array}{c}
b t, \\
a b c d f t, b c d e f t
\end{array} \mid q ; a c d e\right] d_{q} t \\
=\frac{d(1-q)(q, d q / c, c / d, a b c d, b c d e, b c d f, c d e f, a c d f ; q)_{\infty}}{(a c, a d, b c, b d, c e, d e, c f, d f ; q)_{\infty}}
\end{gathered}
$$

Proof: Dividing both sides of (3.1) by $(a b c d, a c d e ; q)_{\infty}$. we obtain

$$
\int_{c}^{d} \frac{(q t / c, q t / d, a b c d e t ; q)_{\infty}}{(a t, b t, e t, a b c d, a c d e ; q)_{\infty}} d_{q} t=\frac{d(1-q)(q, d q / c, c / d, b c d e ; q)_{\infty}}{(a c, a d, b c, b d, c e, d e ; q)_{\infty}}
$$

Taking the action $T\left(f D_{q}\right)$ on both sides of the above identity, we have

$$
\begin{aligned}
& \int_{c}^{d} \frac{(q t / c, q t / d ; q)_{\infty}}{(b t, e t ; q)_{\infty}} T\left(f D_{q}\right)\left\{\frac{(a b c d e t ; q)_{\infty}}{(a t, a b c d, a c d e ;)_{\infty}}\right\} d_{q} t \\
= & \frac{d(1-q)(q, d q / c, c / d, b c d e ; q)_{\infty}}{(b c, b d, c e, d e ; q)_{\infty}} T\left(f D_{q}\right)\left\{\frac{1}{(a c, a d ; q)_{\infty}}\right\}
\end{aligned}
$$

By the Leibniz formula, it follows that

$$
\begin{aligned}
& T\left(f D_{q}\right)\left\{\frac{(a b c d e t ; q)_{\infty}}{(a t, a b c d, a c d e ; q)_{\infty}}\right\} \\
& =\sum_{n=0}^{\infty} \frac{(b t ; q)_{n}(c d e)^{n}}{(q ; q)_{n}} \sum_{k=0}^{\infty} \frac{f^{k}}{(q ; q)_{k}} D_{q}^{k}\left\{\frac{a^{n}}{(a t, a b c d ; q)_{\infty}}\right\} \\
& =\sum_{n=0}^{\infty} \frac{(b t ; q)_{n}(c d e)^{n}}{(q ; q)_{n}} \sum_{k=0}^{\infty} \frac{f^{k}}{(q ; q)_{k}} \sum_{j=0}^{k} q^{j(j-k)}\left[\begin{array}{c}
k \\
j
\end{array}\right] D_{q}^{j}\left\{\frac{1}{(a t, a b c d ; q)_{\infty}}\right\} D_{q}^{k-j}\left(a q^{j}\right)^{n} \\
& =\sum_{n=0}^{\infty} \frac{(b t ; q)_{n}(c d e)^{n}}{(q ; q)_{n}} \sum_{j=0}^{\infty} \frac{\left(f D_{q}\right)^{j}}{(q ; q)_{j}}\left\{\frac{1}{(a t, a b c d ; q)_{\infty}}\right\} \sum_{m=0}^{n} q^{j(n-m)} a^{n-m}\left[\begin{array}{c}
n \\
m
\end{array}\right] f^{m} \\
& =\sum_{n=0}^{\infty} \frac{(b t ; q)_{n}(c d e)^{n}}{(q ; q)_{n}} \sum_{m=0}^{n} a^{n-m}\left[\begin{array}{c}
n \\
m
\end{array}\right] f^{m} T\left(f q^{n-m} D_{q}\left\{\frac{1}{(a t, a b c d ; q)_{\infty}}\right\}\right. \\
& \left.=\sum_{m=0}^{\infty} \frac{(f c d e)^{m}}{(q ; q)_{m}} \sum_{k=0}^{\infty} \frac{(b t ; q)_{k+m}}{(q ; q)_{k}}(a c d e)^{k} \frac{\left(a b c d f t q^{k} ; q\right)_{\infty}}{\left(a t, a b c d, f t q^{k}, b c d f q^{k} ; q\right)_{\infty}}\right\} \\
& =\frac{(a b c d f t ; q)_{\infty}}{(a t, a b c d, f t, b c d f ; q)_{\infty}} \sum_{k=0}^{\infty} \frac{(f t, b c d f, b t ; q)_{k}}{(q, a b c d f t ; q)_{k}}(a c d e)^{k} \sum_{m=0}^{\infty} \frac{\left(q^{k} b t ; q\right)_{m}}{(q ; q)_{m}}(f c d e)^{m} \\
& =\frac{(a b c d f t, b c d e f t ; q)_{\infty}}{(a t, a b c d, f t, b c d f, c d e f ; q)_{\infty}}{ }_{3} \phi_{2}\left[\begin{array}{c}
b t, \quad f t, b c d f \\
a b c d f t, b c d e f t
\end{array} \mid q ; a c d e\right]
\end{aligned}
$$


and

$$
T\left(f D_{q}\right)\left\{\frac{1}{(a c, a d ; q)_{\infty}}\right\}=\frac{(a c d f ; q)_{\infty}}{(a c, a d, c f, d f ; q)_{\infty}}
$$

Combining (3.3) and (3.4), we get Theorem 1.

\section{A generalization of Gasper's Formula}

We observe the following integral formula which was discovered by Gasper ([5]), In ([3]), Chen and Liu had proved it from the Asky-Roy intergral in one step of parameter augmentation.

$$
\begin{gathered}
\frac{1}{2 \pi} \int_{-\pi}^{\pi} \frac{\left(\rho e^{i \theta} / d, q d e^{-i \theta} / \rho, \rho c e^{-i \theta}, q e^{i \theta} / c \rho, a b c d f e^{i \theta} ; q\right)_{\infty}}{\left(a e^{i \theta}, b e^{i \theta}, f e^{i \theta}, c e^{-i \theta}, d e^{-i \theta} ; q\right)_{\infty}} d \theta \\
=\frac{(\rho c / d, d q / \rho c, \rho, q / \rho, a b c d, b c d f, a c d f ; q)_{\infty}}{(q, a c, a d, b c, b d, c f, d f ; q)_{\infty}}
\end{gathered}
$$

where $\max |a|,|b|,|c|,|d|<1, c d \rho \neq 0$.

In this paper, we obtain the following Theorem by again using the q-exponential operator technique on it.

Theorem 4.1. we have

$$
\begin{gathered}
\frac{1}{2 \pi} \int_{-\pi}^{\pi} \frac{\left(\rho e^{i \theta} / d, q d e^{-i \theta} / \rho, \rho c e^{-i \theta}, q e^{i \theta} / c \rho, a b c d f g e^{i \theta}, b c d f g e^{i \theta} ; q\right)_{\infty}}{\left(a e^{i \theta}, b e^{i \theta}, f e^{i \theta}, g e^{i \theta}, c e^{-i \theta}, d e^{-i \theta} ; q\right)_{\infty}} \\
\times_{3} \phi_{2}\left[\begin{array}{r}
f e^{i \theta}, \quad g e^{i \theta}, \quad g c d f \\
a c d f g e^{i \theta}, b c d f g e^{i \theta} \mid
\end{array} \mid q ; a b c d\right] d \theta \\
=\frac{(\rho c / d, d q / \rho c, \rho, q / \rho, a c d f, a c d g, b c d f, b c d g, c d f g ; q)_{\infty}}{(q, a c, a d, b c, b d, c f, d f, c g, d g ; q)_{\infty}}
\end{gathered}
$$

Proof: Dividing both sides of $(4.1)$ by $(a b c d, a c d f ; q)_{\infty}$, and taking the action of $T\left(g D_{q}\right)$ on both sides of it, we obtain

$$
\begin{array}{r}
\frac{1}{2 \pi} \int_{-\pi}^{\pi} \frac{\left(\rho e^{i \theta} / d, q d e^{-i \theta} / \rho, \rho c e^{-i \theta}, q e^{i \theta} / c \rho ; q\right)_{\infty}}{\left(b e^{i \theta}, f e^{i \theta}, c e^{-i \theta}, d e^{-i \theta} ; q\right)_{\infty}} T\left(g D_{q}\right)\left\{\frac{\left(a b c d f e^{i \theta} ; q\right)_{\infty}}{\left(a e^{i \theta}, a b c d, a c d f ; q\right)_{\infty}}\right\} d \theta \\
=\frac{(\rho c / d, d q / \rho c, \rho, q / \rho)_{\infty}}{(q, b c, b d, c f, d f ; q)_{\infty}} T\left(g D_{q}\right)\left\{\frac{1}{(a c, a d ; q)_{\infty}}\right\}
\end{array}
$$


By the Leibniz formula, it follows that

$$
\begin{aligned}
& T\left(g D_{q}\right)\left\{\frac{\left(a b c d f e^{i \theta} ; q\right)_{\infty}}{\left(a e^{i \theta}, a b c d, a c d f ; q\right)_{\infty}}\right\} \\
& =\sum_{n=0}^{\infty} \frac{\left(f e^{i \theta} ; q\right)_{n}(b c d)^{n}}{(q ; q)_{n}} \sum_{k=0}^{\infty} \frac{g^{k}}{(q ; q)_{k}} D_{q}^{k}\left\{\frac{a^{n}}{\left(a e^{i \theta}, a c d f ; q\right)_{\infty}}\right\} \\
& =\sum_{n=0}^{\infty} \frac{\left(f e^{i \theta} ; q\right)_{n}(b c d)^{n}}{(q ; q)_{n}} \sum_{k=0}^{\infty} \frac{g^{k}}{(q ; q)_{k}} \sum_{j=0}^{k} q^{j(j-k)}\left[\begin{array}{c}
k \\
j
\end{array}\right] D_{q}^{j}\left\{\frac{1}{\left(a e^{i \theta}, a c d f ; q\right)_{\infty}}\right\} D_{q}^{k-j}\left(a q^{j}\right)^{n} \\
& =\sum_{n=0}^{\infty} \frac{\left(f e^{i \theta} ; q\right)_{n}(b c d)^{n}}{(q ; q)_{n}} \sum_{j=0}^{\infty} \frac{\left(g D_{q}\right)^{j}}{(q ; q)_{j}}\left\{\frac{1}{\left(a e^{i \theta}, a c d f ; q\right)_{\infty}}\right\} \sum_{m=0}^{n} q^{j(n-m)} a^{n-m}\left[\begin{array}{c}
n \\
m
\end{array}\right] g^{m} \\
& =\sum_{n=0}^{\infty} \frac{\left(f e^{i \theta} ; q\right)_{n}(b c d)^{n}}{(q ; q)_{n}} \sum_{m=0}^{n} a^{n-m}\left[\begin{array}{c}
n \\
m
\end{array}\right] g^{m} T\left(g q^{n-m} D_{q}\left\{\frac{1}{\left(a e^{i \theta}, a c d f ; q\right)_{\infty}}\right\}\right. \\
& \left.=\sum_{m=0}^{\infty} \frac{(g b c d)^{m}}{(q ; q)_{m}} \sum_{k=0}^{\infty} \frac{\left(f e^{i \theta} ; q\right)_{k+m}}{(q ; q)_{k}}(a b c d)^{k} \frac{\left(a c d f g e^{i \theta} q^{k} ; q\right)_{\infty}}{\left(a e^{i \theta}, a c d f, g e^{i \theta} q^{k}, g c d f q^{k} ; q\right)_{\infty}}\right\} \\
& =\frac{\left(a b c d f g e^{i \theta} ; q\right)_{\infty}}{\left(a e^{i \theta}, a c d f, g e^{i \theta}, g c d f ; q\right)_{\infty}} \sum_{k=0}^{\infty} \frac{\left(g e^{i \theta}, g c d f, f e^{i \theta} ; q\right)_{k}}{\left(q, a c d f g e^{i \theta} ; q\right)_{k}}(a b c d)^{k} \sum_{m=0}^{\infty} \frac{\left(q^{k} f e^{i \theta} ; q\right)_{m}}{(q ; q)_{m}}(g b c d)^{m}
\end{aligned}
$$

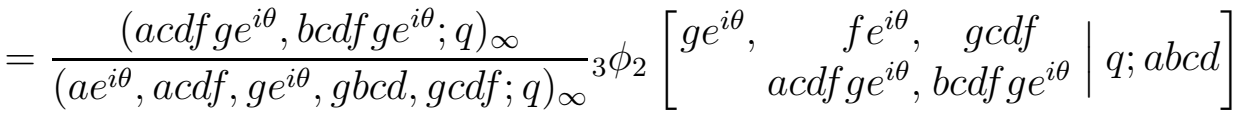

and

$$
T\left(g D_{q}\right)\left\{\frac{1}{(a c, a d ;)_{\infty}}\right\}=\frac{(a c d g ; q)_{\infty}}{(a c, a d, c g, d g ; q)_{\infty}}
$$

Combining (4.3) and (4.4), we get Theorem 2.

\section{References}

[1] S.Roman, More on the umbual calculus, with Emphasis on the q-umbral caculus, J. Math. Anal. Appl. 107 (1985), 222-254.

[2] W. Y. C. chen-Z. G. Liu , Parameter augemntation for basic hypergeometric series I, B. E. Sagan, R. P. Stanley(Eds), Mathematical Essays in honor fo Gian-Carlo Rota, Birkauser, Basel. 1998, pp. 111-129.

[3] W. Y. C. chen-Z. G. Liu, Parameter augemntation for basic hypergeometric series II, Journal of Combinatorial Theory, Series A 80. 1997, 175-195 .

[4] G. Gasper - M. Rahman, Basic Hypergeometric Series (2nd edition), Cambridge University Press, 2004.

[5] G. Gasper , q-Extensions of Barnes', Cauchy's and Euler's beta integrals, "Topics in Mathematical Analysis" (T. M. Rassias. Ed.), pp. 294-314, World Scientific, Singapore, 1989. 